\title{
Single production of leptoquarks at the Fermilab Tevatron
}

\author{
O. J. P. Éboli* and T. L. Lungov ${ }^{\dagger}$ \\ Instituto de Física Teórica - UNESP, Rua Pamplona 145, 01405-900 São Paulo, Brazil
}

(Received 8 November 1999; published 10 March 2000)

\begin{abstract}
We study single production of first generation leptoquarks in association with $e^{ \pm}$at the Fermilab Tevatron. We focus our attention on final states exhibiting an $e^{+} e^{-}$pair and jets, and perform a detailed analysis of the signal and background. The single leptoquark production cross section depends on the leptoquark Yukawa coupling to lepton-quark pairs and we show that the study of this mode can extend considerably the leptoquark search for a large range of these couplings. In fact, for Yukawa couplings of electromagnetic strength, the combined results of the Tevatron experiments can exclude the existence of leptoquarks with masses up to 260-285 (370-425) GeV at the run I (run II), depending on their type.

PACS number(s): 12.60.-i, 13.85.Rm, 14.80.- -
\end{abstract}

\section{INTRODUCTION}

Leptoquarks are an undeniable signal of physics beyond the standard model (SM), and consequently, there have been several direct searches for them in accelerators. In fact, many theories that treat quarks and leptons on the same footing, such as composite models [1,2], technicolor [3], and grand unified theories [4], predict the existence of new particles, called leptoquarks, which mediate quark-lepton transitions. Since leptoquarks couple to a lepton and a quark, they are color triplets under $\mathrm{SU}(3)_{C}$, carry simultaneously lepton and baryon number, have fractional electric charge, and can be of scalar or vector nature.

From the experimental point of view, leptoquarks possess the striking signature of a peak in the invariant mass of a charged lepton with a jet, which make their search rather simple without the need of intricate analyses of several final state topologies. So far, all leptoquark searches have led to negative results. At the hadron colliders, leptoquarks can be pair produced by gluon-gluon and quark-quark fusions, as well as singly produced in association with a lepton in gluonquark reactions. At the Fermilab Tevatron, the CDF and DØ Collaborations studied the pair production of leptoquarks which decay into electron-jet pairs [5]. The combined CDF and $\mathrm{D} \emptyset$ limit on the leptoquark mass is $M_{\mathrm{lq}}>242 \mathrm{GeV}$ [6] for scalar leptoquarks decaying exclusively into $e^{ \pm}$-jet pairs. At the DESY ep collider HERA, first generation leptoquarks are produced in the $s$ channel through their Yukawa couplings, and the HERA experiments [7] placed limits on their masses and couplings, establishing that $M_{l q}$ ₹215-275 GeV depending on the leptoquark type.

In this work we study the single production of first generation leptoquarks $(S)$ in association with a charged lepton at the Tevatron [8], i.e.,

$$
p \bar{p} \rightarrow S e^{ \pm} \rightarrow e^{+} e^{-} \text {jets. }
$$

We perform a careful analysis of all possible QCD and electroweak backgrounds for the topology exhibiting jets plus an

\footnotetext{
*Email address: eboli@ift.unesp.br

${ }^{\dagger}$ Email address: thais@ift.unesp.br
}

$e^{+} e^{-}$pair using the event generator PYTHIA [9]. The signal was also generated using this package. We devised a series of cuts not only to reduce the background, but also to enhance the signal. Since the available phase space for single production is larger than the one for double production, we show that a single leptoquark search can extend considerably the Tevatron bounds on these particles. Our results indicate that the combined results of the Tevatron experiments can exclude the existence of leptoquarks with masses up to 260285 (370-425) GeV at the run I (run II), depending on their type, for Yukawa couplings of electromagnetic strength.

It is interesting to notice that pair production of scalar leptoquarks in a hadronic collider is essentially model independent since the leptoquark-gluon interaction is fixed by the $\mathrm{SU}(3)_{C}$ gauge invariance. On the other hand, single production is model dependent because it takes place via the unknown leptoquark Yukawa interactions. Notwithstanding, these two signals for scalar leptoquarks are complementary because they allow us not only to reveal their existence but also to determine their properties such as mass and Yukawa couplings to quarks and leptons. In this work, we also study the region in parameter space where single leptoquark production can be isolated from pair production.

The outline of this paper is as follows. In Sec. II we introduce the $\mathrm{SU}(3)_{C} \otimes \mathrm{SU}(2)_{L} \otimes \mathrm{U}(1)_{Y}$ invariant effective Lagrangians that we analyze. We also discuss in this section the main features of the leptoquark signal and respective backgrounds. We present our results in Sec. III while our conclusions are drawn in Sec. IV.

\section{LEPTOQUARK SIGNALS AND BACKGROUNDS}

We assume that scalar-leptoquark interactions are $\mathrm{SU}(3)_{C} \otimes \mathrm{SU}(2)_{L} \otimes \mathrm{U}(1)_{Y}$ gauge invariant above the electroweak symmetry breaking scale $v$. Moreover, leptoquarks must interact with a single generation of quarks and leptons with chiral couplings in order to avoid low energy constraints $[10,11]$. The most general effective Lagrangian satisfying these requirements and baryon number $(B)$, lepton number $(L)$, electric charge, and color conservations is [12] 


$$
\begin{aligned}
\mathcal{L}_{e f f}= & \mathcal{L}_{F=2}+\mathcal{L}_{F=0}, \\
\mathcal{L}_{F=2}= & g_{1 L} \bar{q}_{L}^{c} i \tau_{2} l_{L} S_{1 L}+g_{1 R} \bar{u}_{R}^{c} e_{R} S_{1 R}+\tilde{g}_{1 R} \bar{d}_{R}^{c} e_{R} \widetilde{S}_{1} \\
& +g_{3 L} \bar{q}_{L}^{c} i \tau_{2} \vec{\tau} l_{L} \cdot \vec{S}_{3}, \\
\mathcal{L}_{F=0}= & h_{2 L} R_{2 L}^{T} \bar{u}_{R} i \tau_{2} l_{L}+h_{2 R} \bar{q}_{L} e_{R} R_{2 R} \\
& +\widetilde{h}_{2 L} \widetilde{R}_{2}^{T} \bar{d}_{R} i \tau_{2} l_{L},
\end{aligned}
$$

where $F=3 B+L, q(l)$ stands for the left-handed quark (lepton) doublet, and we omitted the flavor indices of the leptoquark couplings to fermions. The leptoquarks $S_{1 R(L)}$ and $\widetilde{S}_{1}$ are singlets under $\mathrm{SU}(2)_{L}$, while $R_{2 R(L)}$ and $\widetilde{R}_{2}$ are doublets, and $S_{3}$ is a triplet.

From the above interactions we can see that first generation leptoquarks can decay into pairs $e^{ \pm} q$ and $\nu_{e} q^{\prime}$, thus giving rise to an $e^{ \pm}$plus a jet or a jet plus missing energy. However, the branching ratio of leptoquarks into these final states depends on the existence of further decays, e.g., into new particles. In this work we considered only the $e^{ \pm} q$ decay mode and that the branching ratio into this channel $(\beta)$ is a free parameter. As we can see from Eqs. (3) and (4), only the leptoquarks $R_{2 L}^{2}, \widetilde{R}_{2}^{2}$, and $S_{3}^{-}$decay exclusively into a jet and a neutrino, and consequently cannot give rise to the topology that we are interested in.

The event generator PYTHIA assumes that the leptoquark interaction with quarks and leptons is described by

$$
\bar{e}\left(a+b \gamma_{5}\right) q
$$

and the leptoquark cross sections are expressed in terms of the parameter $\kappa$ defined as

$$
\kappa \alpha_{\mathrm{em}} \equiv \frac{a^{2}+b^{2}}{4 \pi}
$$

with $\alpha_{\mathrm{em}}$ being the fine structure constant. We present our results in terms of the leptoquark mass $M_{l q}$ and $\kappa$, it being trivial to translate $\kappa$ into the coupling constants appearing in Eqs. (3) and (4); see Table I. The subprocess cross section for the associated production of a leptoquark and a charged lepton,

$$
q+g \rightarrow S+l
$$

depends linearly on the parameter $\kappa$ defined in Eq. (6). For the range of leptoquark masses accessible at the Tevatron, leptoquarks are rather narrow resonances with their width given by

$$
\Gamma(S \rightarrow l q)=\frac{\kappa \alpha_{\mathrm{em}}}{2} M_{\mathrm{lq}}
$$

At the parton level, the single production of leptoquarks leads to a final state exhibiting a pair $e^{+} e^{-}$and $q(\bar{q})$. After the parton shower and hadronization the final state usually contains more than one jet. An interesting feature of the final state topology $e^{+} e^{-}$and jets is that the double production of
TABLE I. Leptoquarks that can be observed through their decays into an $e^{ \pm}$and a jet and the correspondent branching ratios into this channel, assuming that there are no new particles. We also show the relation between the leptoquark Yukawa coupling and the parameter $\kappa$ used in PYTHIA.

\begin{tabular}{lccc}
\hline \hline Leptoquark & Decay & Branching ratio & $4 \pi \alpha_{\mathrm{em}} \kappa$ \\
\hline$S_{1 L}$ & $e^{+} \bar{u}$ & $50 \%$ & $\frac{g_{1 L}^{2}}{2}$ \\
$S_{1 R}$ & $e^{+} \bar{u}$ & $100 \%$ & $\frac{g_{1 R}^{2}}{2}$ \\
$\widetilde{S}_{1 R}$ & $e^{+} \bar{d}$ & $100 \%$ & $\frac{\tilde{g}_{1 R}^{2}}{2}$ \\
$S_{3}^{+}$ & $e^{+} \bar{d}$ & $100 \%$ & $g_{3}^{2}$ \\
$S_{3}^{0}$ & $e^{+} \bar{u}$ & $50 \%$ & $\frac{g_{3}^{2}}{2}$ \\
$R_{2 L}^{1}$ & $e^{-\bar{u}}$ & $100 \%$ & $\frac{h_{2 L}^{2}}{2}$ \\
$R_{2 R}^{1}$ & $e^{-\bar{u}}$ & $100 \%$ & $\frac{h_{2 R}^{2}}{2}$ \\
$R_{2 R}^{2}$ & $e^{-\bar{d}}$ & $100 \%$ & $\frac{h_{2 R}^{2}}{2}$ \\
$\widetilde{R}_{2}^{1}$ & $e^{-\bar{d}}$ & $100 \%$ & $\frac{\tilde{h}_{2 L}^{2}}{2}$ \\
\hline \hline
\end{tabular}

leptoquarks also contributes to it. Consequently, the topology $e^{+} e^{-}$jets has a cross section larger than pair or single leptoquark production alone, increasing the reach of the Tevatron. In principle we can separate single from double production, for instance, by requiring the presence of a single jet in the event. However, in the absence of any leptoquark signal, it is interesting not to impose this cut since in this case the signal cross section gets enhanced, leading to more stringent bounds.

We exhibit in Table II the total cross section for single production of leptoquarks that couple only to $e^{ \pm} u$ or $e^{ \pm} d$ pairs, assuming $\kappa=1$ and $\beta=1$ and requiring one electron with $p_{T}>50 \mathrm{GeV}$, another one with $p_{T}>20 \mathrm{GeV}$, and $|\eta|$ $<4.2$ for both $e^{ \pm}$. In our analyses we used the default proton structure function in PYTHIA 5.7 (CTEQ2L). Notice that the cross sections for single production of $e^{+} q$ and $e^{-} q$ leptoquarks - that is, $|F|=0$ or 2-are equal at the Tevatron. Furthermore, the cross section for single production of a leptoquark coupling only to $u$ quarks is approximately twice the

TABLE II. Total cross section in fb for the single production of a leptoquark that couples only to $l q$ pairs for several leptoquark masses and center-of-mass energies of 1.8/2.0 TeV. We required that one $e^{ \pm}$have $p_{T}>50 \mathrm{GeV}$, the other one $p_{T}>20 \mathrm{GeV}$, and $|\eta|<4.2$ for both $e^{ \pm}$and assumed $\kappa \beta=1$. We indicate by - when the cross section is negligible

\begin{tabular}{lcccc}
\hline \hline$l q$ coupling & $M_{1 \mathrm{q}}=200 \mathrm{GeV}$ & $250 \mathrm{GeV}$ & $300 \mathrm{GeV}$ & $350 \mathrm{GeV}$ \\
\hline$e^{ \pm} u$ & $187 / 259$ & $59 / 86$ & $20 / 30$ & $-/ 12$ \\
$e^{ \pm} d$ & $77 / 112$ & $22 / 34$ & $7 / 11$ & $-/ 4$ \\
\hline \hline
\end{tabular}


TABLE III. Total cross section in fb for the pair production leptoquarks that couple only to $l q$ pairs for several leptoquark masses and center-of-mass energies of 1.8/2.0 TeV. We imposed the same cuts as in Table II.

\begin{tabular}{lcccc}
\hline \hline$l q$ coupling & $M_{\mathrm{lq}}=200 \mathrm{GeV}$ & $250 \mathrm{GeV}$ & $300 \mathrm{GeV}$ & $350 \mathrm{GeV}$ \\
\hline$e^{ \pm} u$ & $153 / 237$ & $30.9 / 53.1$ & $6.6 / 13.0$ & $-/ 3.23$ \\
$e^{ \pm} d$ & $153 / 225$ & $29 / 50.1$ & $6.2 / 12.0$ & $-/ 3.00$ \\
\hline \hline
\end{tabular}

one for leptoquarks coupling only to $d$ quarks, in agreement with a naive valence-quark-counting rule. We display in Table III the production cross section of leptoquark pairs for the same choice of the parameters and cuts used in Table II. The small difference between the cross sections for the production of $e^{ \pm} u$ and $e^{ \pm} d$ leptoquarks is due to the exchange of an $e^{ \pm}$in the $t$ channel of the reaction $q \bar{q} \rightarrow S \bar{S}$.

In our analyses we kept track of the $e^{ \pm}$(jet) carrying the largest transverse momentum, which we denote by $e_{1}\left(j_{1}\right)$, and the $e^{ \pm}$(jet) with the second largest $p_{T}$, which we called $e_{2}\left(j_{2}\right)$. The reconstruction of the jets in the final state was done using the subroutine LUCELL of PYTHIA, requiring the transverse energy of the jet to be larger than $7 \mathrm{GeV}$ inside a cone $\Delta R=\sqrt{\Delta \eta^{2}+\Delta \phi^{2}}=0.7$.

The transverse momentum distributions of the $e_{1}$ and $j_{1}$ originating from leptoquarks are shown in Fig. 1, where we required that $p_{T}^{e_{1}}>50 \mathrm{GeV}, p_{T}^{e_{2}}>20 \mathrm{GeV}$, and $|\eta|<4.2$ for both $e^{ \pm}$. In this figure, we added the contributions from single and pair production of $u e^{-}$leptoquarks of mass $M_{\mathrm{lq}}$ $=300 \mathrm{GeV}$ for $\sqrt{s}=2.0 \mathrm{TeV}$. We can see from this figure that the $e_{1}$ and $j_{1}$ spectra are peaked approximately at $M_{\mathrm{lq}} / 2$ and exhibit a large fraction of hard leptons, and consequently the $p_{T}$ cut on $e_{1}$ does not reduce significantly the signal.

Within the scope of the SM, there are many sources of backgrounds leading to jets accompanied by a pair $e^{+} e^{-}$. We divided them into three classes [13].

$Q C D$ processes. The reactions included in the QCD class are initiated by hard scatterings proceeding exclusively through the strong interaction. In this class of processes, the main source of hard $e^{ \pm}$is the semileptonic decay of hadrons possessing quarks $c$ or $b$, which are produced in the hard scattering or in the parton shower through the splitting $g$ $\rightarrow c \bar{c}(b \bar{b})$. Important features of the events in this class are that close to the hard $e^{ \pm}$there is a substantial amount of

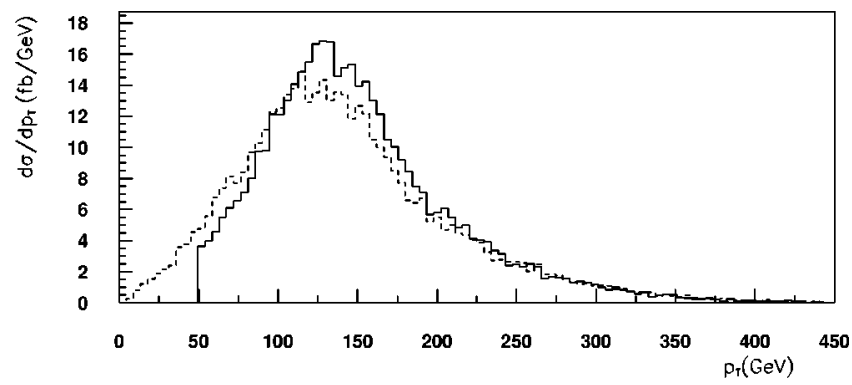

FIG. 1. $p_{T}$ spectrum of the largest transverse momentum $e^{ \pm}$(solid line) and jet (dashed line). We added single and double production of $u e^{+}$leptoquarks with mass $M_{\mathrm{lq}}=300 \mathrm{GeV}$ for $\sqrt{s}=2.0 \mathrm{TeV}, \kappa=1$, and $\beta=1$.
TABLE IV. Total cross section in $\mathrm{fb}$ of the different background classes for center-of-mass energies of 1.8 and $2.0 \mathrm{TeV}$. We required one $e^{ \pm}$with $p_{T}>50 \mathrm{GeV}$ and the other $e^{\mp}$ with $p_{T}>20 \mathrm{GeV}$. We also demanded that the invariant mass of $e^{+} e^{-}$pair differ from the $Z$ mass by more than $5 \mathrm{GeV}$.

\begin{tabular}{lcc}
\hline \hline Class & $\sigma_{\text {total }}(1.8 \mathrm{TeV})(\mathrm{fb})$ & $\sigma_{\text {total }}(2.0 \mathrm{TeV})(\mathrm{fb})$ \\
\hline QCD & 67 & 129 \\
Electroweak & 453 & 562 \\
Top & 3.9 & 52 \\
\hline \hline
\end{tabular}

hadronic activity and that the $e^{ \pm}$transverse momentum spectrum is peaked at small values.

Electroweak processes. This class contains the Drell-Yan production of quark or lepton pairs and single and pair production of electroweak gauge bosons. It is interesting to notice that the main backgrounds by far in this class are $q_{i} g\left(\bar{q}_{i} \rightarrow Z q_{i}(g)\right.$. This suggests that we should veto events where the invariant mass of the $e^{+} e^{-}$pair is around the $Z$ mass; however, even after such a cut, these backgrounds remain important due to the production of off-shell Z's.

Top quark production. The production of top quark pairs takes place through quark-quark and gluon-gluon fusions. In general, the $e^{ \pm}$produced in the leptonic top quark decay into $b e \nu_{e}$ are rather isolated and energetic. Fortunately, the top quark production cross section at the Tevatron is rather small.

As an illustration, we present in Table IV the total cross section of the above background classes requiring the events to exhibit an $e^{ \pm}$with $p_{T}>50 \mathrm{GeV}$ and a second $e^{\mp}$ having $p_{T}>20 \mathrm{GeV}$ with the invariant mass of this pair differing from the $Z$ mass by more than $5 \mathrm{GeV}$. As we can see from this table, the introduction of this $p_{T}$ cut already reduces the QCD backgrounds to a level below the electroweak processes without on-mass-shell production of Z's. As we naively expect, the increase in the center-of-mass energy has a great impact in the top quark production cross section.

\section{RESULTS}

Taking into account the features of the signal and backgrounds, we imposed the following set of cuts.

(C1) We required the events to exhibit a pair $e^{+} e^{-}$and one or more jets.

(C2) We introduced typical acceptance cuts - that is, the $e^{ \pm}$are required to be in the rapidity region $\left|\eta_{e}\right|<2.5$ and the jet(s) in the region $\left|\eta_{j}\right|<4.2$.

(C3) One of the $e^{ \pm}$should have $p_{T}>50 \mathrm{GeV}$ and the other $p_{T}>20 \mathrm{GeV}$.

(C4) The $e^{ \pm}$should be isolated from hadronic activity. We required that the transverse energy deposited in a cone of size $\Delta R=0.5$ around the $e^{ \pm}$direction to be smaller than 10 $\mathrm{GeV}$.

(C5) We rejected events where the invariant mass of the pair $e^{+} e^{-}\left(M_{e_{1} e_{2}}\right)$ is close to the $Z$ mass; i.e., we demanded that $\left|M_{e_{1} e_{2}}-M_{Z}\right|<30 \mathrm{GeV}$. This cut reduces the backgrounds coming from $Z$ decays into a pair $e^{+} e^{-}$. 


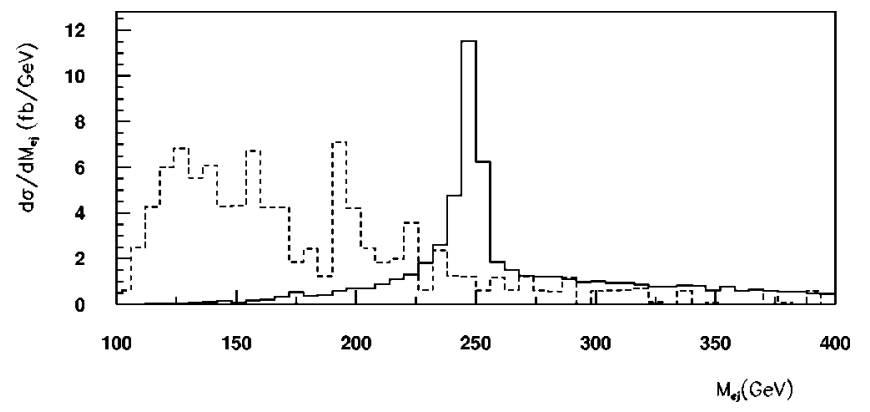

FIG. 2. $M_{e j}$ spectrum due to the background (dashed line) and a leptoquark of mass $250 \mathrm{GeV}$ with $\kappa=1$ and $\beta=1$ (solid line) after the cuts (C1)-(C6) are applied for $\sqrt{s}=1.8 \mathrm{TeV}$.

(C6) We required that all the invariant masses $M_{e_{i} j_{k}}(i$, $k=1,2)$ be larger than $10 \mathrm{GeV}$.

(C7) We accepted only the events which exhibit a pair $e^{ \pm}$ jet with an invariant mass $M_{e j}$ in the range $\left|M_{e j}-M_{\mathrm{lq}}\right|$ $<30 \mathrm{GeV}$. An excess of events signals the production of a leptoquark.

In Fig. 2 we present the $M_{e j}$ spectrum after the cuts (C1)(C6) originating from the background and the production of an $e^{+} u$ leptoquark of mass $250 \mathrm{GeV}$ with $\kappa=\beta=1$. The largest invariant mass of the four possible combinations is plotted both for background (dashed line) and signal (solid line). The signal peak is clearly seen out of the background.

\section{A. Pair production}

At this point it is interesting to obtain the attainable bounds on leptoquarks springing from the search of leptoquark pairs. In this case we required, in addition to cuts (C1) $-(\mathrm{C} 7)$, that the events present two $e^{ \pm}$-jet pairs with invariant masses satisfying $\left|M_{e j}-M_{\mathrm{lq}}\right|<30 \mathrm{GeV}$. Our results show that $\mathrm{CDF}$ and $\mathrm{D} \emptyset$ should be able to constrain the lep-

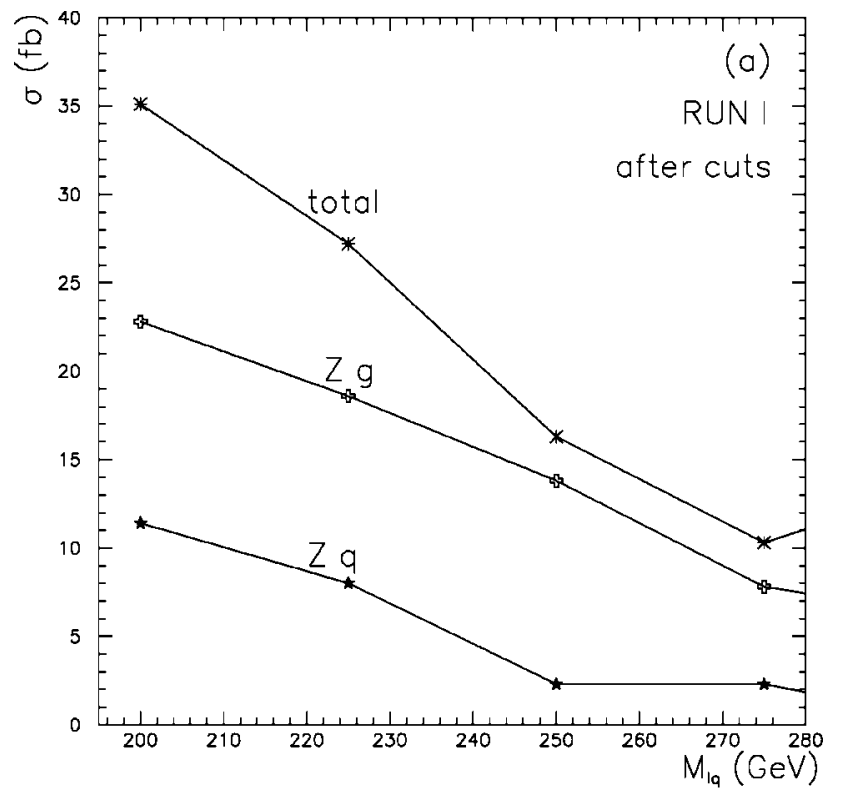

toquark masses to be heavier than $225(350) \mathrm{GeV}$ at the run I (run II) for $\beta=1$ and $\kappa=0$, assuming that only the background is observed. When the data of both experiment are combined, the limit changes to 250 (375) GeV. It is interesting to notice that our results for run I are compatible with the ones obtained by the Tevatron collaborations. Moreover, taking into account the single production of leptoquarks changes these constraints only by a few $\mathrm{GeV}$ for $\kappa=1$.

\section{B. Single production}

We display in Fig. 3 the total background cross section and its main contributions as a function of $M_{l q}$ after applying the cuts $(\mathrm{C} 1)-(\mathrm{C} 7)$ for center-of-mass energies of 1.8 and 2.0 $\mathrm{TeV}$. We can see from this figure that the number of expected background events per experiment at run I (II) is 4 (102) for $M_{\mathrm{lq}}=200 \mathrm{GeV}$, dropping to 0 (8) for $M_{\mathrm{lq}}$ $=400 \mathrm{GeV}$. For the sake of comparison, we display in Fig. 4 the total cross section for the production of $e^{+} u$ and $e^{-} d$ leptoquarks assuming $\kappa=1$ and $\beta=1$ for the same cuts and center-of-mass energies.

We estimated the capability of the Tevatron to exclude regions of the plane $\kappa \beta \otimes M_{\mathrm{lq}}$, assuming that only the background events were observed. We present in Fig. 5(a) the projected 95\% C.L. exclusion region for $e^{+} u$ and $e^{+} d$ leptoquarks at run I with an integrated luminosity of $110 \mathrm{pb}^{-1}$ per experiment. From our results we can learn that the search for single $e^{ \pm} u\left(e^{ \pm} d\right)$ leptoquarks in each experiment can exclude leptoquark masses up to 265 (245) $\mathrm{GeV}$ for $\kappa \beta=1$. Combining the results of $\mathrm{CDF}$ and $\mathrm{D} \emptyset$ expands this range of excluded masses to 285 (260) GeV, respectively. The corresponding results for run II with an integrated luminosity of $2 \mathrm{fb}^{-1}$ per experiment are presented in Fig. 5(b). Here we can see that the combined $\mathrm{CDF}$ and $\mathrm{D} \emptyset$ data will allow us to rule out $e^{ \pm} u\left(e^{ \pm} d\right)$ leptoquarks with masses up to 425 (370) $\mathrm{GeV}$, assuming that $\beta=\kappa=1$.

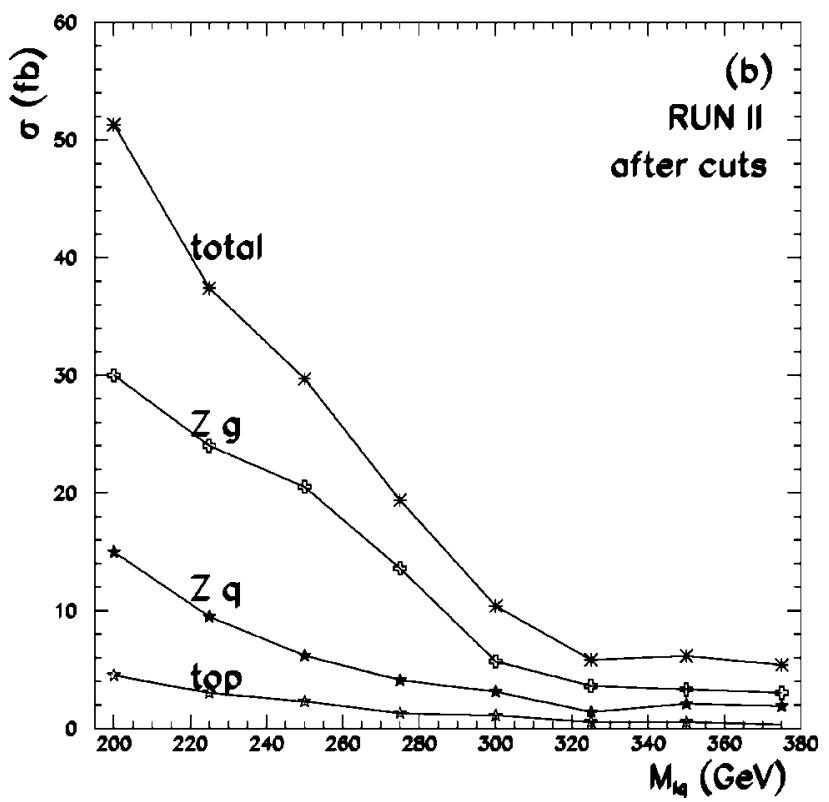

FIG. 3. Total cross sections of the main backgrounds after cuts for (a) $\sqrt{s}=1.8$ and (b) $2.0 \mathrm{TeV}$. The line labeled $Z g$ (Zq) stands for the reaction $q \bar{q}$ $\rightarrow Z g \quad(q g \rightarrow Z q)$ while the line marked top represents the cross section for the production of top quark pairs. 

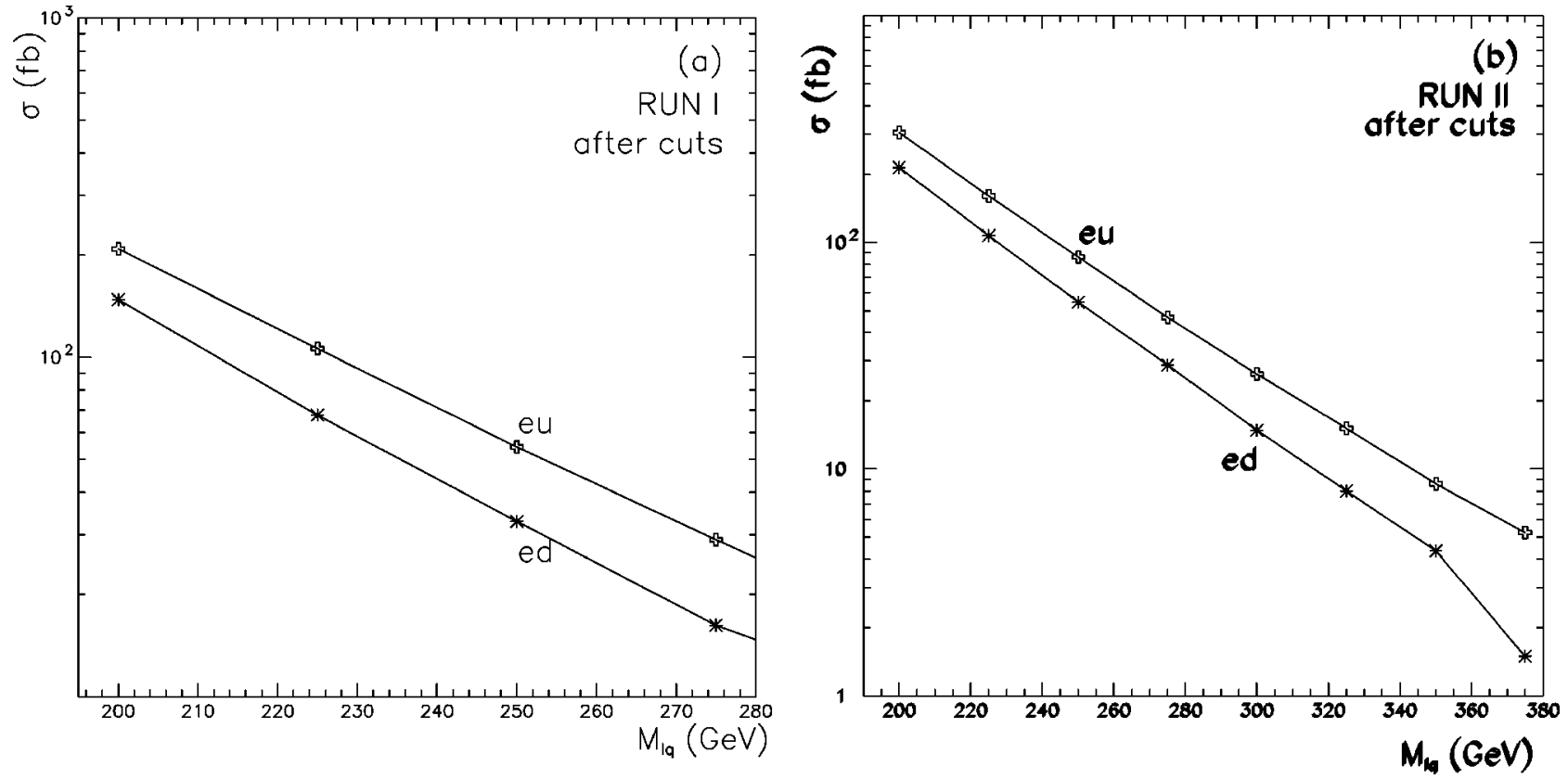

FIG. 4. Total cross sections for the production of $e^{+} u$ and $e^{+} d$ leptoquarks after cuts for (a) $\sqrt{s}=1.8$ and (b) $2.0 \mathrm{TeV}$. We assumed $\kappa=1$ and $\beta=1$.

It is important to stress that events exhibiting a pair of leptoquarks also contribute to our single leptoquark search. This is the reason why lighter leptoquarks can be observed even for arbitrarily small $\kappa$; see Fig. 5. However, the maximum mass that can be excluded for $\kappa=0$ is smaller than the limit coming from the search for leptoquark pairs since the requirement of an additional $e^{ \pm}$-jet pair with invariant mass close to $M_{\mathrm{lq}}$ helps to further reduce the backgrounds. For instance the single leptoquark search can rule out leptoquarks with masses up to $330 \mathrm{GeV}$ for $\kappa=0$ at run II while the search for leptoquark pairs leads to a lower bound of 375 $\mathrm{GeV}$.

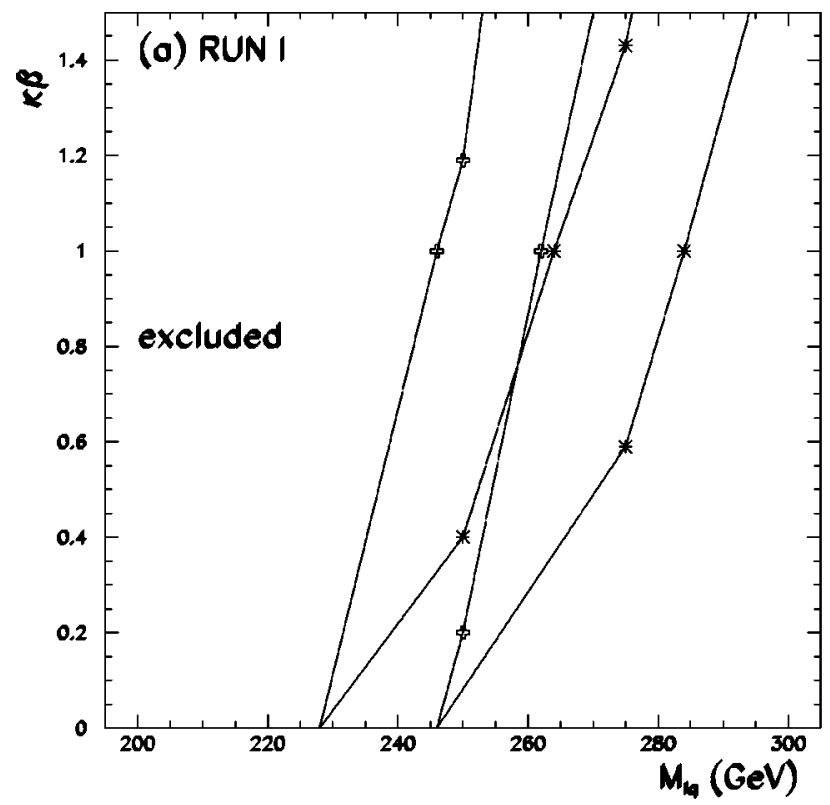

Since the Tevatron can look for quite heavy leptoquarks, the fraction of the (anti)proton momentum carried by the quarks and gluons is rather large. In order to assess the uncertainties in our analyses coming from the choice of proton structure function, we obtained the $\mathrm{CDF}$ and $\mathrm{D} \emptyset$ combined limits coming from single production of $e^{ \pm} u$ and $e^{ \pm} d$ leptoquarks using the CTEQ4HJ parton distribution function [14]. We found out that the Tevatron will rule out $e^{ \pm} u$ and $e^{ \pm} d$ leptoquarks with masses up to 412 and $362 \mathrm{GeV}$. This represents a decrease in the reach of the single leptoquark search of the order of $13 \mathrm{GeV}$. However, this channel still has a much larger reach than the search for leptoquark pairs.

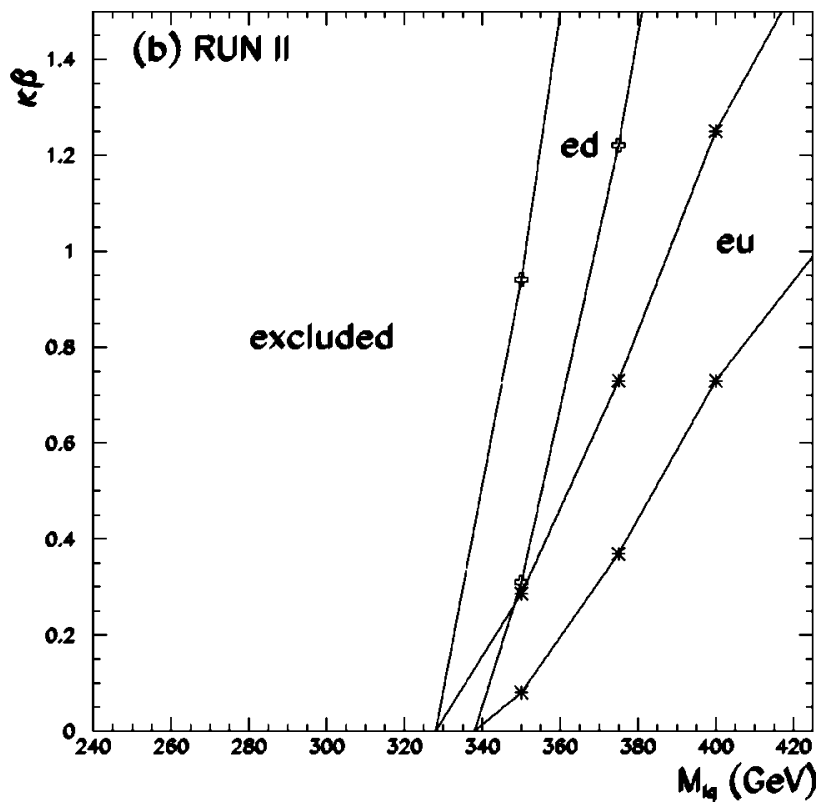

FIG. 5. 95\% C.L. excluded region in the $\kappa \beta \otimes M_{\mathrm{lq}}$ for (a) $\sqrt{s}=1.8$ and (b) $2.0 \mathrm{TeV}$. The curves with crosses (stars) correspond to the bounds on $e^{ \pm} d$ $\left(e^{ \pm} u\right)$ leptoquarks, with the upper (lower) one originating from the results of a single (combined) experiment(s). 


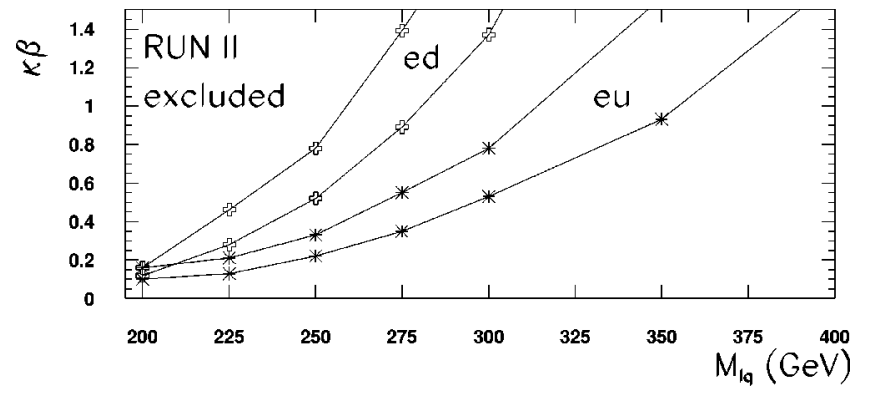

FIG. 6. 95\% C.L. excluded region in the $\kappa \beta \otimes M_{\mathrm{lq}}$ for $\sqrt{s}=2.0 \mathrm{TeV}$ when we impose cuts $(\mathrm{C} 1)-(\mathrm{C} 7)$ and also demand that the events exhibit just one jet. The curves with crosses (stars) correspond to the bounds on $e^{ \pm} d\left(e^{ \pm} u\right)$ leptoquarks, with the upper (lower) one originating from the results of a single (combined) experiment(s).

In principle we can separate the double production of leptoquarks from the single one. An efficient way to extract the single leptoquark events is to require that just one jet be observed. At run I this search leads to an observable effect only for rather large values of $\kappa$. On the other hand, this search can be done at run II; however, the bounds coming from this analysis are weaker than the ones obtained above; see Fig. 6. We can interpret this figure as the region of the $\kappa \beta \otimes M_{\mathrm{lq}}$ where we can isolate single leptoquark production and study this process in detail.

\section{CONCLUSIONS}

The analyses of the single production of leptoquarks at the Tevatron run I allow us to extend the range of excluded masses beyond the present limits stemming from the search of leptoquark pairs. We showed that in the absence of any excess of events CDF and $\mathrm{D} \emptyset$ individually should be able to probe $e^{ \pm} u\left(e^{ \pm} d\right)$ leptoquark masses up to 265 (245) $\mathrm{GeV}$ for Yukawa couplings of the electromagnetic strength and $\beta=1$. In the case $\beta=0.5$ these limits reduce to 250 (235) $\mathrm{GeV}$. Moreover, combining the results from both experiments can further increase the Tevatron reach for leptoquarks. Assuming that leptoquarks decay exclusively into the known quarks and leptons and $\kappa=1$, the combined Tevatron results can exclude $S_{1 L}$ and $S_{3}^{0}$ leptoquarks with masses up to $270 \mathrm{GeV}, S_{1 R}, R_{2 L}^{1}$, and $R_{2 R}^{1}$ leptoquarks with masses 285 $\mathrm{GeV}$, and $\widetilde{S}_{1 R}, S_{3}^{+}, R_{2 R}^{2}$, and $\widetilde{R}_{2}^{1}$ with masses up to 260 $\mathrm{GeV}$. These results represent an improvement over the present bounds obtained at the Tevatron [5]; however, the bounds are similar to the ones obtained by the HERA Collaborations [7].

At run II, the search for the single production of leptoquarks will be able to rule out leptoquarks with masses even larger. For instance, the $\mathrm{CDF}$ and $\mathrm{D} \emptyset$ combined results can probe $e^{ \pm} u$ ( $e^{ \pm} d$ ) leptoquark masses up to 425 (370) $\mathrm{GeV}$ for $\kappa \beta=1$. In the case $\kappa \beta=0.5$, these bounds reduce to 385 (350) $\mathrm{GeV}$. However, even these improved limits will not reach the level of the indirect bounds ensuing from low energy physics $[10,11,15]$. Direct limits more stringent than indirect ones will only be available at the $\operatorname{LHC}[13,16]$ or future $e^{+} e^{-}$colliders [17].

\section{ACKNOWLEDGMENTS}

This work was supported by Conselho Nacional de Desenvolvimento Científico e Tecnológico (CNPq), by Fundação de Amparo à Pesquisa do Estado de São Paulo (FAPESP), and by Programa de Apoio a Núcleos de Excelência (PRONEX).
[1] For a review see, W. Buchmüller, Acta Phys. Austriaca, Suppl. 27, 517 (1985).

[2] L. Abbott and E. Farhi, Nucl. Phys. B189, 547 (1981).

[3] S. Dimopoulos, Nucl. Phys. B168, 69 (1981); E. Farhi and L. Susskind, Phys. Rev. D 20, 3404 (1979); J. Ellis et al., Nucl. Phys. B182, 529 (1981).

[4] See, for instance, P. Langacker, Phys. Rep. 72, 185 (1981); J. L. Hewett and T. G. Rizzo, ibid. 183, 193 (1989).

[5] CDF Collaboration, F. Abe et al., Phys. Rev. D 48, 3939 (1993); Phys. Rev. Lett. 75, 1012 (1995); D $\emptyset$ Collaboration, S. Abachi et al., Phys. Rev. Lett. 72, 965 (1994); 75, 3618 (1995); CDF Collaboration, F. Abe et al., ibid. 79, 4327 (1997); CDF Collaboration, F. Abe et al., ibid. 78, 2906 (1997); DØ Collaboration, B. Abbott et al., ibid. 79, 4321 (1997); 80, 2051 (1998).

[6] CDF Collaboration and DØ Collaboration, C. Grosso-Pilcher et al., Report No. FERMILAB-PUB-98-312-E, hep-ex/9810015, presented at 1996 DPF/DPB Summer Study on New Directions for High-Energy Physics (Snowmass '96), Snowmass, CO, 1996.

[7] ZEUS Collaboration, M. Derrick et al., Phys. Lett. B 306, 173
(1993); H1 Collaboration, I. Abt et al., Nucl. Phys. B396, 3 (1993); H1 Collaboration, S. Aid et al., Phys. Lett. B 369, 173 (1996); H1 Collaboration, C. Adloff et al., Z. Phys. C 74, 191 (1997); ZEUS Collaboration, J. Breitweg et al., ibid. 74, 207 (1997).

[8] J. L. Hewett and S. Pakvasa, Phys. Rev. D 37, 3165 (1988); O. J. P. Éboli and A. V. Olinto, ibid. 38, 3461 (1988); B. Dion, L. Marteau, and G. Simon, ibid. 56, 479 (1997).

[9] T. Sjöstrand, Comput. Phys. Commun. 82, 74 (1994).

[10] O. Shanker, Nucl. Phys. B204, 375 (1982).

[11] W. Buchmüller and D. Wyler, Phys. Lett. B 177, 377 (1986); J. C. Pati and A. Salam, Phys. Rev. D 10, 275 (1974).

[12] W. Buchmüller, R. Rückl, and D. Wyler, Phys. Lett. B 191, 442 (1987).

[13] O. J. P. Éboli, R. Z. Funchal, and T. L. Lungov, Phys. Rev. D 57, 1715 (1998).

[14] H. L. Lai et al., Phys. Rev. D 55, 1280 (1997).

[15] S. Davidson, D. Bailey, and A. Campbell, Z. Phys. C 61, 613 (1994); G. Bhattacharyya, J. Ellis, and K. Sridhar, Phys. Lett. B 336, 100 (1994); 338, 522(E) (1994); O. J. P. Éboli, M. C. Gonzalez-Garcia, and J. K. Mizukoshi, Nucl. Phys. B443, 20 
(1995); M. Leurer, Phys. Rev. Lett. 71, 1324 (1993); Phys. Rev. D 49, 333 (1994).

[16] J. Ohnemus, S. Rudaz, T. F. Walsh, and P. Zerwas, Phys. Lett. B 334, 203 (1994); J. E. Cieza Montalvo and O. J. P. Éboli, Phys. Rev. D 50, 331 (1994); J. Blümlein, E. Boos, and A. Krykov, Z. Phys. C 76, 137 (1997); M. Krämer et al., Phys. Rev. Lett. 79, 341 (1997); J. L. Hewett and T. Rizzo, Phys. Rev. D 56, 5709 (1997); M. S. Berger and W. Merritt, hep-ph/9611386; J. E. Cieza Montalvo et al., Phys. Rev. D 58, 095001 (1998); B. Dion et al., Eur. Phys. J. C 2, 497 (1998); B. Dion, L. Marleau, and G. Simon, Phys. Rev. D 59, 015001 (1999).
[17] J. L. Hewett and T. G. Rizzo, Phys. Rev. D 36, 3367 (1987); J. L. Hewett and S. Pakvasa, Phys. Lett. B 227, 178 (1987); J. E. Cieza Montalvo and O. J. P. Éboli, Phys. Rev. D 47, 837 (1993); J. Blümein and R. Rückl, Phys. Lett. B 304, 337 (1993); J. Blümlein, E. Boos, and A. Krykov, ibid. 392, 150 (1997); M. S. Berger, hep-ph/9609517; F. Cuypers, P. H. Frampton, and R. Rueckl, Phys. Lett. B 390, 221 (1997); O. J. P. Éboli et al., ibid. 311, 147 (1993); H. Nadeau and D. London, Phys. Rev. D 47, 3742 (1993); M. A. Doncheski and S. Godfrey, ibid. 51, 1040 (1995); T. M. Aliev, E. Iltan, and N. K. Pak, ibid. 54, 4263 (1996); M. A. Doncheski and S. Godfrey, hep-ph/9612385 (Snowmass '96); G. Bélanger, D. London, and H. Nadeau, Phys. Rev. D 49, 3140 (1994). 